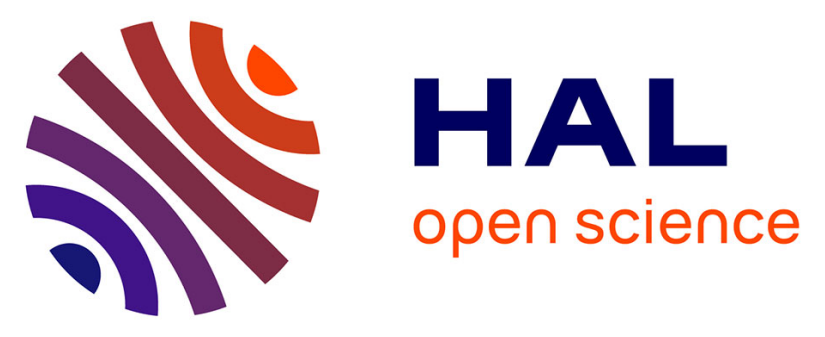

\title{
Thresholding-based distributed power control for energy-efficient interference networks
}

\author{
Chao Zhang, Achal Agrawal, Vineeth Varma, Samson Lasaulce
}

\section{To cite this version:}

Chao Zhang, Achal Agrawal, Vineeth Varma, Samson Lasaulce. Thresholding-based distributed power control for energy-efficient interference networks. 29th Annual IEEE International Symposium on Personal, Indoor and Mobile Radio Communications, PIMRC 2018, Sep 2018, Bologna, Italy. 10.1109/pimrc.2018.8580827 . hal-01943045

\section{HAL Id: hal-01943045 \\ https://hal.science/hal-01943045}

Submitted on 3 Dec 2018

HAL is a multi-disciplinary open access archive for the deposit and dissemination of scientific research documents, whether they are published or not. The documents may come from teaching and research institutions in France or abroad, or from public or private research centers.
L'archive ouverte pluridisciplinaire HAL, est destinée au dépôt et à la diffusion de documents scientifiques de niveau recherche, publiés ou non, émanant des établissements d'enseignement et de recherche français ou étrangers, des laboratoires publics ou privés. 


\title{
Thresholding-based distributed power control for energy-efficient interference networks
}

\author{
Chao ZHANG*, Achal AGRAWAL ${ }^{\ddagger}$, Vineeth S VARMA ${ }^{\dagger}$, and Samson LASAULCE* \\ * L2S (CNRS-CentraleSupelec-Univ. Paris Sud), Gif-sur-Yvette, France \\ ¥ Mahindra Ecole Centrale, Hyderabad, India \\ ${ }^{\dagger}$ Université de Lorraine, CNRS, CRAN, 54000 Nancy, France
}

\begin{abstract}
In this paper, we propose simple one-shot power control functions and assess their performance both through analytical and numerical results. The proposed functions only assume individual channel state information (CSI) at each transmitter and are based on channel inversion and more importantly on thresholding; a transmitter uses zero power if the channel gain is below a threshold. Although the idea of thresholding has been used for maximizing spectral efficiency, it has not been used for maximizing (the total network) energy-efficiency (EE), which is measured here in terms of sum-EE. More specifically, we prove the optimality of the proposed policy in asymptotic regimes such as the low and high interference scenarios. We also prove that the expected sum-energy is individually quasi-concave with respect to each of the thresholds; this allows us to provide a lowcomplexity algorithm which can be run offline to find good thresholds. Through numerical simulations, we show that the simple idea of thresholding provides very appreciable gains.
\end{abstract}

\section{INTRODUCTION}

The design of energy-efficient communications is an issue that has gained a lot of attention in the past years. This issue is naturally relevant in mobile devices with limited battery capacity, but it has also become an important issue for radio base stations and tele-communication operators due to the operational costs [1]. In [2], an extensive survey is provided on techniques that improve energy efficiency in wireless systems, defined as the ratio between the number of bits successfully transmitted and the power consumed. Amongst the various scenarios that can be considered, the focus of this paper, is on transmission power control. Ideally, to optimize power control in distributed interference networks, a transmitter requires knowledge about global channel state information (CSI). However, in a distributed setting, global CSI is typically not available. The existing techniques to acquire global CSI, typically rely on the existence of inter-transmitter signaling channels (see [3]), which may be unavailable in practice. Some recent works have proposed techniques with which global CSI can be estimated using implicit signalling through the interference channel via power modulation [4]. However, these works assume a sufficiently long channel coherence time as global channel acquisition may take a long time. Additionally, the technique proposed does not scale well with a large number of interfering devices. In the present work, we only look at fully distributed power control policies that: provide the transmit power level in one shot that is, without generating extra delay induced by memory reading or exchanges with the backhauling infrastructure; only exploit individual CSI as available information; may be used even in the presence of many (interfering) users.

Energy-efficient distributed power control in the sense adopted in the present paper has been first studied in [5], in which the problem of power control is treated as a non-cooperative game with each transmitting device attempts to maximize its individual energy-efficiency. Various extensions, see [6]-[10] have been proposed to [5], in which additional features like multi-band channels, OFDMA, MIMO, packet layer, relays, etc., was considered. However, the resulting operating point is at the Nash equilibrium which can be seen to be very inefficient in terms of sum-EE. This work was later improved in [11] in which a pricing function is artificially added to the individual utility in order to incite cooperative behavior in multiple access channels (MAC). While the resulting operating point Pareto dominates the equilibrium from [5], it is still not designed to be optimal and more importantly, the operating point can not be determined as an explicit function of the channel gain. However, not many works propose a technique that optimizes or improves upon the sum-EE considered in [11], in the context of an interference channel, which is the primary motivation of this work. To our knowledge, the closest work is given by [12] in which a thresholding policy was shown to be near-optimal for sum-rate maximization.

The main advantages and features of the proposed technique over the state of the art can be summarized as follows.

1) It is designed for interference channels and not only multiple access channels (as done in [11] and is therefore more general.

2) Once the thresholds are determined offline, the power control at each time instant can be determined in a shot manner and by an explicit 
function. This means without an iterative online procedure which might not converge and necessitate several and possibly many SINR samples (as in [11] and papers using the best-response dynamics), especially when the number of users increases.

3) It is optimal when the CSI is individual and in extreme regimes of interference level.

This paper is structured as follows. In Section II we provide the system model and problem statement, followed by an analysis of the proposed thresholding policy in. We then show some properties of this policy in IV which facilitates in tuning the threshold. In Section $\mathrm{V}$ we compare the energy efficiency obtained with the proposed technique with state of the art techniques and provide some concluding remarks in VI.

\section{PROBLEM FORMULATION}

Consider a wireless network where $K$ transmitters (or transmitter-receiver pairs depending on the model considered) are trying to coordinate the power emitted $p_{i} \in \mathcal{P}_{i}, i \in \mathcal{K}=\{1, \ldots, K\}$. Due to the power constraint at every transmitter, the transmit power at any transmitter $i \in \mathcal{K}$ should not exceed $P_{\max }$, i.e. $p_{i} \in\left[0, P_{\max }\right]$. Based on the transmission power profile, the signal to interference plus noise ratio (SINR) at each receiver $i$ can be written as

$$
\gamma_{i}=\frac{p_{i} g_{i i}}{\sigma^{2}+\sum_{j=1, j \neq i}^{K} p_{j} g_{j i}}
$$

where the channel gain coefficient $g_{j i} \in \mathbb{R}_{\geqslant 0}$ represents the quality of wireless link from the transmitter $j$ to the receiver $i$, and $\sigma^{2}$ represents the noise level. We define the global channel state $G=\left(g_{11}, \ldots, g_{K K}\right) \in \mathbb{R}^{K^{2}}$, which is in general, a random state which affects the common payoff function for the system, and is not controlled by the coordinating transmitters. The realizations of the channel gain coefficients $G$ at each time instant are assumed to be i.i.d. and follow a probability distribution $\rho$. In this work, $\rho$ is taken to be an exponential distribution for each channel gain $g_{i j}$, i.e. we consider a Rayleigh fading channel.

The instantaneous individual utility function $u_{i}\left(G, p_{1}, \ldots, p_{N}\right)$, is the energy efficiency as defined in [5], i.e.,

$$
u_{i}\left(G, p_{1}, \ldots, p_{K}\right)=\frac{\psi\left(\gamma_{i}\right)}{p_{i}}
$$

where $\psi$ denotes the expected number of successful bits transmitted as a function of the SINR; $\psi$ may represent the packet success rate and is typically a sigmoidal function. The sum- $E E$ is therefore $w\left(G, p_{1}, \ldots, p_{K}\right)=$ $\sum_{i=1}^{K} u_{i}\left(G, p_{1}, \ldots, p_{K}\right)$. In this work, we are interested in maximizing the expected sum-EE defined as

$\mathbb{E}_{G}\left[w\left(G, p_{1}, \ldots, p_{K}\right)\right]=\int_{G \in \mathcal{G}} w\left(G, p_{1}, \ldots, p_{N}\right) \rho(G) \mathrm{d} G$
Since only individual CSI, i.e. , $g_{i i}$ is available at $i$, the optimal power control must be tuned based solely on $g_{i i}$. Although in general, this policy might also depend on past values of the channel, we focus on stationary strategies and our objective is to find an optimal mapping $f_{i}\left(g_{i i}\right): \mathbb{R}_{\geqslant 0} \rightarrow\left[0, P_{\max }\right]$. This induces no performance loss as the nature state is considered to be i.i.d. at each time. We use $f:=\left(f_{1}(\cdot), \ldots, f_{K}(\cdot)\right)$ to denote the global power control policy and $\mathcal{F}$ to denote the set of all such functions. In general, the optimization problem to be solved can be written as

$$
\operatorname{Maximize}_{f \in \mathcal{F}} \mathbb{E}_{G}\left[w\left(G, p_{1}, \ldots, p_{K}\right)\right] .
$$

However, optimizing the above functional is not trivial at all in general, and we therefore restrict our family of functions to the following type of thresholding policies,

$$
f_{\lambda_{i}}\left(g_{i i}\right)= \begin{cases}0 & \text { if } g_{i i}<\lambda_{i} \\ \frac{\alpha_{i} \sigma^{2}}{g_{i i}} & \text { if } g_{i i} \geqslant \lambda_{i}\end{cases}
$$

where $\lambda_{i} \geqslant 0$ is the threshold for Transmitter $i$ and $\alpha_{i} \geqslant 0$ is a scaling factor to be tuned. For instance, in [5], choosing $\alpha_{i}$ to be the unique positive solution of $\gamma \psi^{\prime}(\gamma)-\psi(\gamma)=0$, i.e., $\alpha_{i}=\gamma^{\star}$, is shown to correspond to the Nash equilibrium solution. This is the choice we will make but other choices are possible.

\section{ANALYSIS}

Extensive numerical simulations for the case with discrete alphabets for $\mathcal{G}$, i.e., when $|\mathcal{G}|<\infty$, revealed the structure of the optimal solution to be of the form (5). These simulations involved an exhaustive search over all possible mappings $f_{i}(\cdot)$ (which is finite) [13]. Conjecturing a similar continuous power control policy from observations on the discrete case was not aberrant as taking the limit of the alphabet size growing larger brings the system close to the continuous case.

While we have no proof for the optimality of the proposed power control policy (5) in the general case, for asymptotic cases of small and large interference, it can be shown that such a policy is optimal. This is precisely the objective of proposition III.1, wherein we consider the two asymptotic cases of very high and negligible interference. To quantify these terms, we introduce signal-to-interference ratio (SIR) as a measure for high interference and low interference respectively:

$$
\begin{aligned}
& \mathrm{SIR}_{\text {max }}=\max _{i, j, j \neq i}\left(\frac{\mathbb{E}\left(g_{i i}\right)}{\mathbb{E}\left(g_{j i}\right)}\right) \\
& \mathrm{SIR}_{\text {min }}=\min _{i, j, j \neq i}\left(\frac{\mathbb{E}\left(g_{i i}\right)}{\mathbb{E}\left(g_{j i}\right)}\right)
\end{aligned}
$$

Since the power control policy is a function of $g_{i i}$, we must find the optimal power for each value of given 
$g_{i i}$. In the following proposition, we prove that when $\mathrm{SIR}_{\text {max }} \rightarrow 0$ (high interference) or $\mathrm{SIR}_{\text {min }} \rightarrow \infty$ (low interference), the optimal power control policy is of the form (5). For ease of exposition, we assume the case where the power limitation is always met, i.e. $P_{\max } \rightarrow$ $\infty$. For finite $P_{\max }$ the power control can be taken as the min of $P_{\max }$ and the one provided below.

Proposition III.1. When $\mathrm{SIR}_{\max } \rightarrow 0$ or $\mathrm{SIR}_{\min } \rightarrow \infty$, the optimization problem (4) has a solution of the form (5).

\section{Proof:}

First of all, if we denote the interference of user $i$ as

$$
I_{i}=\sum_{j \neq i} g_{j i} p_{j}
$$

knowing that $g_{j i}$ follows the exponential distribution (as assumed in Section II), we have

$$
\lim _{\mathbb{E}\left(g_{j i}\right) \rightarrow \infty} \operatorname{Pr}\left(g_{j i}>\frac{1}{\epsilon}\right)=1
$$

for any $\epsilon \in \mathbb{R}_{>0}$, i.e., $g_{j i} \rightarrow \infty$ almost surely in the low SIR limit. Similarly,

$$
\lim _{\mathbb{E}\left(g_{j i}\right) \rightarrow \infty} \operatorname{Pr}\left(g_{j i}<\epsilon\right)=1
$$

which means that $g_{j i} \rightarrow 0$ almost surely in the high SIR limit.

When $\mathrm{SIR}_{\text {min }} \rightarrow \infty$, since the interference is negligible here, each user $i$ will choose the action which maximizes its own utility, leading to the optimal solution $f_{i}^{*}\left(g_{i i}\right)=\frac{\gamma^{\star} \sigma^{2}}{g_{i i}}$.

When $\operatorname{SIR}_{\text {max }} \rightarrow 0$, we can make some relevant observations. First, if $p_{j}>0$, then $\frac{\psi\left(\gamma_{i}\right)}{p_{i}} \rightarrow 0$ for all $i \neq j$ regardless of $p_{i}$ since $g_{j i} \rightarrow \infty$ and $\gamma_{i} \rightarrow 0$. Therefore, if the right transmitter $j$ is chosen, then $p_{j}^{*}>0, p_{i}^{*}=0$. Naturally, since $j$ experiences no interference, $p_{j}^{*}=\frac{\gamma^{\star} \sigma^{2}}{g_{j j}}$. This lets us conclude that in the low SIR limit, the optimal power control policy if done in a centralized manner would be to pick the best transmitter (largest $g_{j j}$ ) and switch off all the others.

For a given transmitter $i$, since individual CSI is available, we must try to optimize the energy efficiency function over all possible realizations of the other gains, i.e. let $G_{-i}=\left(g_{11}, g_{12}, \ldots, g_{i, i-1}, g_{i, i+1}, \ldots, g_{K K}\right)$ denote all the other gains and $\rho_{-i}\left(G_{-i} \mid g_{i i}\right)$ denote the p.d.f. of the other gains. Then (3) can be rewritten as:

$$
\begin{aligned}
& \mathbb{E}_{G}[w(G, P)] \\
= & \int_{G \in \mathcal{G}} w\left(G, f_{1}\left(g_{11}\right), \ldots, f_{K}\left(g_{K K}\right)\right) \rho(G) \mathrm{d} G \\
= & \int_{g_{i i}} \rho_{i}\left(g_{i i}\right) \int_{G_{-i}} w\left(G, \ldots, f_{K}\left(g_{K K}\right)\right) \rho_{-i}\left(G_{-i} \mid g_{i i}\right) \mathrm{d} G \\
= & \int_{g_{i i}} \rho_{i}\left(g_{i i}\right) \theta_{i}\left(g_{i i}, f_{i}\left(g_{i i}\right)\right) \mathrm{d} g_{i i}
\end{aligned}
$$

where

$$
\begin{aligned}
& \theta_{i}\left(g_{i i}, f_{i}\left(g_{i i}\right)\right) \\
= & \int_{G_{-i}} w\left(G, f_{1}\left(g_{11}\right), \ldots, f_{K}\left(g_{K K}\right)\right) \rho_{-i}\left(G_{-i} \mid g_{i i}\right) \mathrm{d} G_{-i}
\end{aligned}
$$

Since $g_{i j} \rightarrow \infty$ almost surely for all $i \neq j$, when $I_{i}=0$ for some $i$, we have

$$
w\left(G, \ldots, f_{i}\left(g_{i i}\right)=w_{i}(G, \ldots), \ldots\right)=\frac{\psi\left(\frac{g_{i i} f_{i}\left(g_{i i}\right)}{\sigma^{2}}\right)}{f_{i}\left(g_{i i}\right)}
$$

and otherwise, $w\left(G, \ldots, f_{i}\left(g_{i i}\right) \rightarrow 0\right.$.

Hence, from (13) we can conclude that $f_{i}\left(g_{i i}\right)=$ $\frac{\gamma^{\star} \sigma^{2}}{g_{i i}}$ dominates other positive actions almost surely. The optimal action for user $i$ thus belongs to the set $\left\{0, \frac{\gamma^{\star} \sigma^{2}}{g_{i i}}\right\}$.

Next, we prove that there exists an unique threshold between the two actions. Define the difference between the utility of 2 actions as:

$\Delta\left(g_{i i}\right)=\theta_{i}\left(g_{i i}, f_{i}\left(g_{i i}\right)=0\right)-\theta_{i}\left(g_{i i}, f_{i}\left(g_{i i}\right)=\frac{\gamma^{\star} \sigma^{2}}{g_{i i}}\right)$

Then three following conditions can be easily checked:

$$
\begin{gathered}
\frac{\partial \Delta\left(g_{i i}\right)}{g_{i i}}<0 \quad \forall g_{i i}>0 \\
\lim _{g_{i i} \rightarrow 0} \Delta\left(g_{i i}\right)>0 \\
\lim _{g_{i i} \rightarrow \infty} \Delta\left(g_{i i}\right)<0
\end{gathered}
$$

Note that $\Delta\left(g_{i i}\right)$ is a continuous and monotonic function. Hence, we can conclude that there exists one unique threshold $\lambda_{i}$ such that $\Delta\left(g_{i i}=\lambda_{i}\right)=0$, $\Delta\left(g_{i i}\right)>0$ if $g_{i i}<\lambda_{i}$ and $\Delta\left(g_{i i}\right)<0$ if $g_{i i}>\lambda_{i}$ using the mean value theorem.

When the SIR is not in these limits, the threshold policy of the form (5) can not be proven to be optimal. However, through numerical simulations in section $\mathrm{V}$, we shall show that it outperforms the current state of the art on distributed energy efficient power control. While the propositions proved in this section give us some justification for the proposed power control policy, they do not provide a way to compute the optimal thresholds for each transmitter in a practical scenario. In the next section, we shall prove that the expected sumenergy is quasi-concave w.r.t. each individual threshold when the other thresholds are held a constant. This helps us provide a low-complexity distributed algorithm for finding the thresholds for each transmitter, thereby determining their power control policy through equation (5). 


\section{DETERMINING OPTIMAL THRESHOLDS}

We shall now proceed to show that if the power control policy is of the form proposed (5), then the payoff is quasi-concave w.r.t. any individual threshold $\lambda_{i}$ if the other thresholds $\lambda_{j}, j \neq i$ are held a constant. In fact, we can even get a closed form expression for the optimal threshold for a transmitter assuming the thresholds for other transmitters are known. For this, we first define

$$
\begin{aligned}
& \theta_{i}\left(g_{i i}, p_{i} ; \lambda_{-i}\right) \\
= & \int_{G_{-i}} w\left(G, f_{\lambda_{1}}\left(g_{11}\right), . ., p_{i}, . ., f_{\lambda_{K}}\left(g_{K K}\right)\right) \\
& \times \rho_{-i}\left(G_{-i} \mid g_{i i}\right) \mathrm{d} G_{-i}
\end{aligned}
$$

which is the expected sum energy efficiency when transmitter $i$ has channel gain $g_{i i}$ and uses power $p_{i}$, with the rest of the transmitters using the policy (5) with $\lambda_{-i}$ denoting the thresholds of all the other transmitters $j \neq i$.

Proposition IV.1. Assuming $p_{i}=f_{\lambda_{i}}\left(g_{i i}\right)$ as defined in (5), then the expected payoff is quasi-concave w.r.t. the individual threshold $\lambda_{i}$ when the other thresholds are held constant.

Proof. Similar to the proof of Proposition III.1, the expected payoff (3) can be written as:

$$
\begin{aligned}
& \mathbb{E}_{\rho}\left[w\left(G, f_{1}\left(g_{11}\right), \ldots, f_{K}\left(g_{K K}\right)\right)\right] \\
= & \int_{0}^{\lambda_{i}} \rho_{i}\left(g_{i i}\right) \theta_{i}\left(g_{i i}, 0 ; \lambda_{-i}\right) \mathrm{d} g_{i i} \\
& +\int_{\lambda_{i}}^{\infty} \rho_{i}\left(g_{i i}\right) \theta_{i}\left(g_{i i}, \frac{\gamma^{\star} \sigma^{2}}{g_{i i}} \lambda_{-i}\right) \mathrm{d} g_{i i} .
\end{aligned}
$$

Note that when $p_{i}=0, \theta_{i}\left(g_{i i}, 0 ; \lambda_{-i}\right)$ becomes a constant which is independent of $g_{i i}$, i.e., $\theta_{i}\left(g_{i i}, 0 ; \lambda_{-i}\right)=c_{i}$. When $f_{i}\left(g_{i i}\right)=\frac{\gamma^{\star} \sigma^{2}}{g_{i i}}$, define

$$
a_{i}\left(g_{i i}\right)=\theta_{i}\left(g_{i i}, \frac{\gamma^{\star} \sigma^{2}}{g_{i i}} ; \lambda_{-i}\right) .
$$

We can easily verify that

$$
\begin{gathered}
\frac{\partial a_{i}}{\partial g_{i i}}>0 \quad \forall g_{i i}>0, \\
\lim _{g_{i i} \rightarrow 0} a_{i}\left(g_{i i}\right) \leqslant c_{i}, \\
\lim _{g_{i i} \rightarrow \infty} a_{i}\left(g_{i i}\right)>c_{i} .
\end{gathered}
$$

Therefore, it can be concluded that under the assumption of proposed threshold policy, the expected payoff $\mathbb{E}_{\rho}[w(G, P)]$ is increasing w.r.t. $\lambda_{i}$ when $\lambda_{i} \leqslant a_{i}^{-1}\left(c_{i}\right)$ and decreasing w.r.t. $\lambda_{i}$ when $\lambda_{i}>a_{i}^{-1}\left(c_{i}\right)$. The optimum $\lambda_{i}$ to maximize the $\mathbb{E}_{\rho}[w(G, P)]$ can be written as

$$
\lambda_{i}^{\star}=a_{i}^{-1}\left(c_{i}\right)
$$

Thus it can be seen that for every real number $a$,

$$
C_{\lambda_{i}}^{+}=\left\{\lambda_{i} \in \mathcal{R}^{+}: \omega_{i}\left(\lambda_{i}\right) \geqslant a\right\}
$$

is a convex set, which proves our claim about quasiconcavity.

Even though, we have obtained a closed form expression for optimal individual thresholds, this result is not easily exploitable as finding the inverse of the function $a_{i}($.$) is not easy in general.$

Nonetheless, we can use the quasi-concavity property to propose an iterative algorithm to find optimal threshold for each transmitter. An efficient distributed manner to find the individual thresholds $\lambda_{i}, \forall i \in\{1, \ldots, K\}$ would be to perform best response dynamics with each transmitter updating its threshold which maximizes the expected sum-utility given the other thresholds (functions). To find the expected sum-utility for a given $\lambda_{i}$, we evaluate equation (19) numerically through montecarlo simulations. It is important to note that this procedure is performed offline, and the functions found are one-shot decision functions which could be exploited straightaway online.

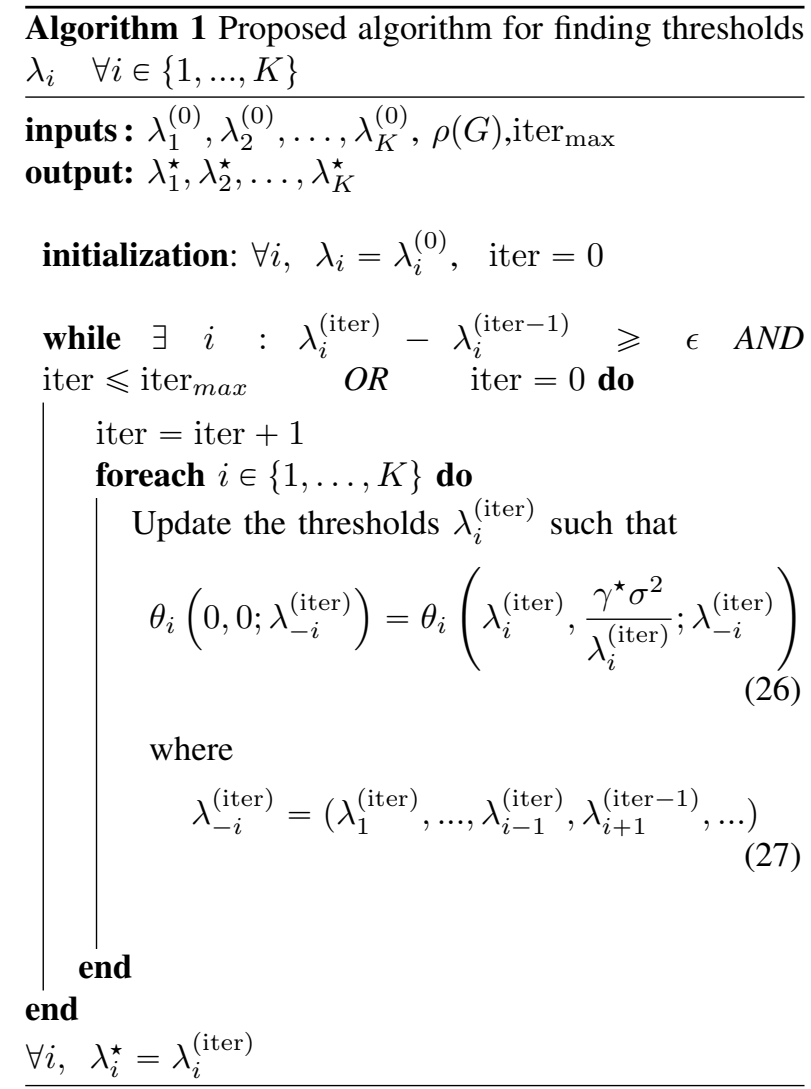

In the next section, we show that our approach brings considerable benefits in terms of sum energy efficiency when compared to state of the art solutions. 


\section{NUMERICAL ANALYSis}

For the simulation setup, we choose typical efficiency function and model parameters. The efficiency function is chosen to be [11]:

$$
f\left(\gamma_{i}\right)=\left(1-e^{-0.5 \gamma_{i}}\right)^{M}
$$

where we also choose the total number of bits per frame $M=80$. For this chosen utility function, we can obtain $\gamma^{\star}=12.4$ by solving $\gamma f^{\prime}(\gamma)=f(\gamma)$. In all the simulations, we choose $P_{\max }=1 \mathrm{~W}$ and $\sigma^{2}=1 \mathrm{~mW}$ which corresponds to a moderate Signal to Noise Ratio (SNR) of $30 \mathrm{~dB}$.

We compare the proposed threshold policy to two important works for team power control problem:

- The solution in [14]: Their algorithm is the closest to the proposed policy in terms of information structure assumed to choose the power level.

- Pricing NE from [11]: Their algorithm requires is more demanding in terms of information as they assume transmitters receiving SINR feedback (possible to recover global CSI $G$ [4]), and that for multiple time slots. The proposed equilibrium can only be achieved by an online best-response dynamics (exploration phase) in addition to an offline optimization of the pricing parameter, while our policy has a closed form expression and can be implemented in one shot. Despite these differences, their algorithm is still distributed in terms of information and decision and is considered a reference in cooperative team power control.

In Fig. 1, we consider two user case with different power control policies. From Fig. 1 we see that the proposed policy, unsurprisingly, outperforms [14] by a big margin. Thresholding indeed helps reduce interference as when individual channels are bad, transmitters do not emit at all. In the case of [14], at lower channel gains, transmitters emit at higher powers thus generating interference.

The Pricing NE performs well by reducing the interference through penalties on higher power emitted. As seen from our simulations, this can be an effective way of containing interference. As mentioned before, their solution however requires time to converge which creates a missed opportunity cost. If one considers the block fading model, i.e. the channel gains remaining constant for a block of $T$ time-slots, if $\mathrm{T}$ is small, the average payoff over all time-slots for pricing solution would be considerably smaller than the results indicated in Fig. 1.

We illustrate the extent of this loss through Fig. 2 where we consider blocks of different sizes $t \in$ $\{1, \ldots, T\}$. We run the pricing NE algorithm and plot the average payoff for $t$ slots where the channel remains constant. Taking this into account, we see that for blocks of small sizes our algorithm performs much better since it chooses the 'correct' power levels right from the beginning as opposed to pricing NE which takes time to converge.

To demonstrate the scalability of the threshold policy, in Fig. 3 we plot the average EE against the number of Transmitter-Receiver pairs. We see similar performance results and trends as in Fig. 1 for the same aforementioned reasons. It can be also seen that threshold policy has relatively higher gains than [14] for large systems since the higher interference can be effectively mitigated by using threshold. However, fig. 3 shows that the gap between our policy and pricing NE becomes more significant when we have more transmitterreceiver pairs. Indeed, in that case information about the realization of others' channels become important for coordination. SINR feedback helps transmitters to share some information about the channel realizations. For threshold policy however, the individual channel realizations cannot be exchanged, and therefore its performance suffers slightly when compared to [11] .

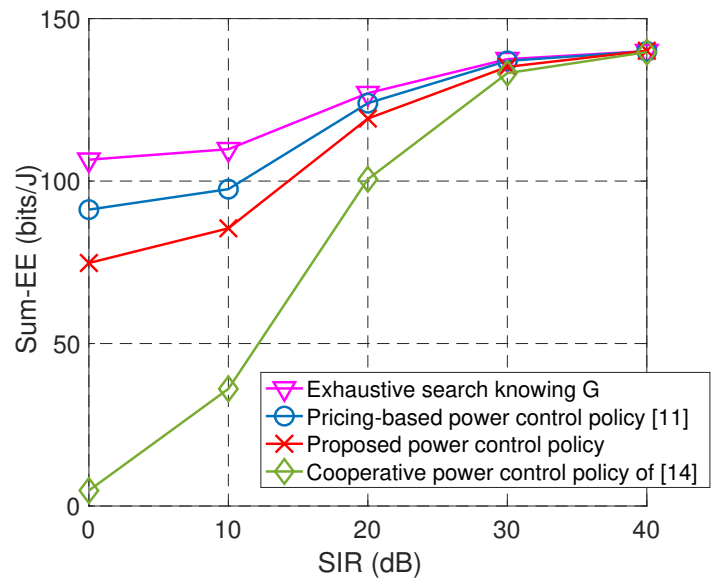

Fig. 1: Comparison of our proposed power control policy with state of the art policies in terms of the resulting sum energy efficiency. Note that the proposed policy outperforms [14] significantly, and is in fact close to [11], despite requiring no online procedure for converging to the equilibrium.

\section{CONCLUSION}

The key takeaway point of our investigations is that thresholding is a powerful tool to naturally incorporate almost-optimal coordination with only individual CSI feedback required to operate. For such simple information structure, our proposed solution gives considerably higher payoff. The fact that it performs almost as well as Pricing NE which requires much more information and preferably stable channel gains is indeed surprising and promising. More analytical results regarding the 


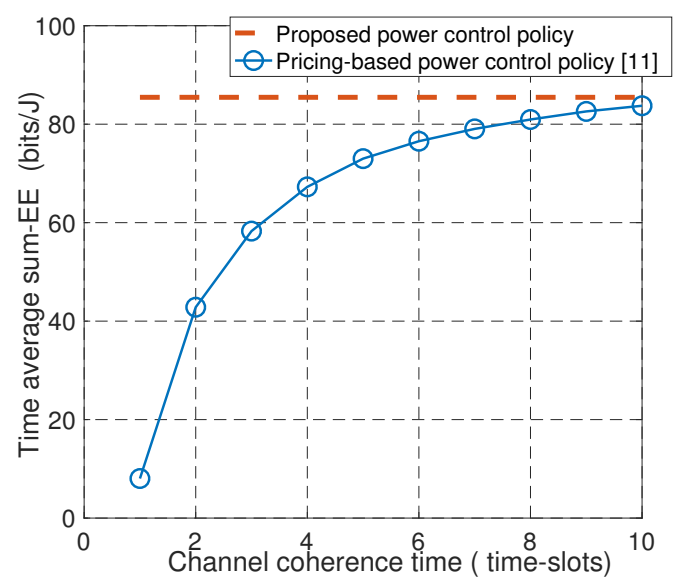

Fig. 2: In this figure, we compare the average energy efficiency as a function of the channel coherence time (in units of time-slots). As this time increases, the online best response algorithm in [11] is able to converge to the equilibrium resulting in a higher average efficiency. However, when the coherence window is small (as in many mobile applications), our proposed policy has a better performance as the power control is determined in one shot.

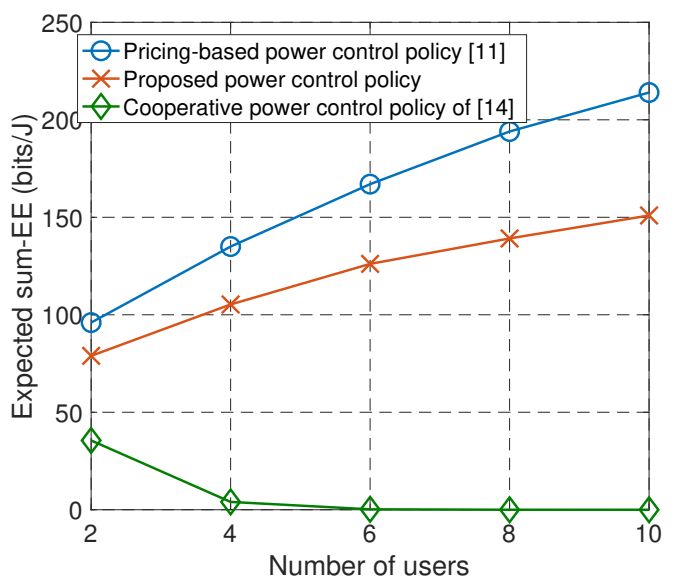

Fig. 3: While other one-shot algorithms like [5] or [14] scale poorly with the number of transmitting devices, the proposed algorithm scales well. The performance is similar to [11] which requires some time online before converging to its equilibrium.

optimality of threshold policies are needed to provide a firmer grounding for our conjecture.

A natural extension of our work would be to conjecture similar strategies for more general problems like power allocation and MIMO. If the results for Power Control are anything to go by, it is well worth investigating.

\section{ACKNOWLEDGEMENT}

This work was funded by the RTE Chair.

\section{REFERENCES}

[1] E. Hossain, V. Bhargava, and G. Fettweis, Green radio communication networks. Cambridge University Press, 2012.

[2] T. Rault, A. Bouabdallah, and Y. Challal, "Energy efficiency in wireless sensor networks: A top-down survey," Computer Networks, vol. 67, pp. 104-122, 2014.

[3] A. Gjendemsjø, D. Gesbert, G. E. Oien, and S. G. Kiani, "Binary power control for sum rate maximization over multiple interfering links," IEEE Transactions on Wireless Communications, vol. 7, no. 8, pp. 3164-3173, 2008.

[4] C. Zhang, V. S. Varma, S. Lasaulce, and R. Visoz, "Interference coordination via power domain channel estimation," IEEE Transactions on Wireless Communications, vol. 16, no. 10, pp. 6779-6794, 2017.

[5] D. Goodman and N. Mandayam, "Power control for wireless data," IEEE Personal Communications, vol. 7, no. 2, pp. 48 54, 2000.

[6] F. Meshkati, M. Chiang, H. V. Poor, and S. C. Schwartz, "A game-theoretic approach to energy-efficient power control in multicarrier CDMA systems," IEEE Journal on Selected Areas in Communications, vol. 24 no. 6, pp. 1115-1129, 2006. [Online]. Available: http://dx.doi.org/10.1109/JSAC.2005.864028

[7] S. Buzzi, G. Colavolpe, D. Saturnino, and A. Zappone, "Potential games for energy-efficient power control and subcarrier allocation in uplink multicell OFDMA systems," J. Sel. Topics Signal Processing, vol. 6, no. 2, pp. 89-103, 2012. [Online]. Available: http://dx.doi.org/10.1109/JSTSP.2011.2177069

[8] E. V. Belmega and S. Lasaulce, "Energy-efficient precoding for multiple-antenna terminals," IEEE Trans. Signal Processing, vol. 59, no. 1, pp. 329-340, 2011. [Online]. Available: http://dx.doi.org/10.1109/TSP.2010.2086451

[9] V. S. Varma, S. Lasaulce, Y. Hayel, and S. E. Elayoubi, "A cross-layer approach for distributed energy-efficient power control in interference networks," IEEE Transactions on Vehicular Technology, vol. 64, no. 7, pp. 3218-3232, 2015.

[10] A. Zappone, S. Buzzi, and E. Jorswieck, "Energy-efficient power control and receiver design in relay-assisted ds/cdma wireless networks via game theory," IEEE Communications Letters, vol. 15, no. 7, pp. 701-703, 2011.

[11] C. U. Saraydar, N. B. Mandayam, and D. J. Goodman, "Efficient power control via pricing in wireless data networks," IEEE transactions on Communications, vol. 50, no. 2, pp. 291-303, 2002.

[12] P. De Kerret, S. Lasaulce, D. Gesbert, and U. Salim, "Bestresponse team power control for the interference channel with local CSI," in Communications (ICC), 2015 IEEE International Conference on. IEEE, 2015, pp. 4132-4136.

[13] C. Zhang, S. Lasaulce, A. Agrawal, and R. Visoz, "A framework for distributed power control with partial channel state information," https://hal.archives-ouvertes.fr/hal-01221639/document.

[14] A. Zappone, Z. Chong, E. A. Jorswieck, and S. Buzzi, "Energyaware competitive power control in relay-assisted interference wireless networks," IEEE Transactions on Wireless Communications, vol. 12, no. 4, pp. 1860-1871, 2013. 